\title{
Quantitative Assessment of the Probability of Bluetongue Virus OVERWINTERING by HORIZONTAL TrANSMISSION IN VeCtORS, Ruminants, OR IN BOTH: ApPLICATION to Germany
}

\author{
S. Napp ${ }^{1 *}$ S. Gubbins ${ }^{2}$ P. Calistri ${ }^{3}$ A. Allepuz ${ }^{1}$ A. Alba ${ }^{1}$ \\ I. García-Bocanegraa ${ }^{1}$ A. Giovannini ${ }^{3}$ J. Casal $^{1,4}$
}

Even though bluetongue virus (BTV) transmission is apparently interrupted during winter, bluetongue outbreaks often reappear in the next season (overwintering). Several mechanisms for BTV overwintering have been proposed, but to date their relative importance remains unclear. In order to assess the probability of BTV overwintering by horizontal transmission in vectors (longlived Culicoides), ruminants (prolonged viraemia), or a combination of both, a quantitative risk assessment model was developed. Furthermore, the model allowed the role played by the residual number of vectors present during winter to be examined, and the effect of a proportion of Culicoides living inside buildings (endophilic behaviour) to be explored. The model was then applied to a real scenario: overwintering in Germany between

2006 and 2007. The results showed that the limited number of vectors active during winter seemed to allow the transmission of BTV during this period and that this transmission was favoured by the endophilic behaviour of some Culicoides. Even though transmission was possible, the likelihood of BTV overwintering by horizontal transmission in vectors, ruminants or in both seemed however too low to explain the observed re-emergence of the disease. Therefore, other overwintering mechanisms that were not considered in the model are likely to have played a significant role in BTV overwintering in Germany between 2006 and 2007.

KEYWords: Culicoides - BluetONGUe - DiSEASE TRANSMISSION - RUMINANT - GERMANY.

1. Centre de Recerca en Sanitat Animal, Unidad de Epidemiología, Campus UAB,

08193 Bellaterra, Barcelona, Spain.

2. Institute for Animal Health, Pirbright, United Kingdom.

3. Istituto Zooprofilattico Sperimentale dell'Abruzzo e del Molise "G. Caporale",

Italy.

4. Departament de Sanitat i Anatomia Animals, UAB, Barcelona, Spain.

* Corresponding author

Tel.: +349358144 57; Fax: +34935814490

E-mail: sebastian.napp@cresa.uab.es 